The Open Dentistry Journal
CrossMark
Content list available at: www.benthamopen.com/TODENTJ/
DOI: $10.2174 / 1874210601610010001$

\title{
Lateral Ridge Augmentation with Autogenous Bone Harvested Using Trephine Drills: A Noninvasive Technique
}

\author{
H. R. Arab ${ }^{1}$, A. Moeintaghavi ${ }^{2, *}$, M. Taheri ${ }^{1}$, N. Sargolzaie ${ }^{3}$, D. Aghasizadeh ${ }^{4}$ and F. Shiezadeh ${ }^{5}$ \\ ${ }^{1}$ Dental Research Center, Mashhad University of Medical Sciences, Iran \\ ${ }^{2}$ Maxillofacial Disease Center, Mashhad University of Medical Sciences, Iran \\ ${ }^{3}$ Dental Material Research Center, Mashhad University of Medical Sciences, Iran \\ ${ }^{4}$ Dental School, Mashhad University of Medical Sciences, Iran \\ ${ }^{5}$ Department of Periodontics, North Khorasan University of Medical Sciences, Bojnurd, Iran
}

\begin{abstract}
:
Purpose:

The aim of this pilot study was to evaluate the success rate of a chairside ridge augmentation procedure using bone autografts harvested with trephine drills and placed without the use of screws.

Methods:

Thirty patients were recruited for the study. After the surgical site was anesthetized and a crestal incision was made, an envelope flap was retracted using blunt dissection limited to the graft site, and the periosteum was raised intact and undamaged from the bone. The flap was extended laterally to obtain sufficient space for the bone graft. At the donor site, bone was obtained from the external oblique ridge area. A \#5 or \#6 trephine drill was used to harvest one or two pieces of bone. The bone blocks were placed inside the envelope flap at the recipient site, which was then sutured and covered with periodontal dressing. Antibiotics, analgesics, and mouthwash were prescribed. Measurements of ridge width were performed using CBCT before and 3 months after surgery. The preand post operative results were compared using paired $t$ test.
\end{abstract}

\section{Results:}

Pre- and post-operative mean ridge widths were $2.23 \pm 0.79$ and $5.16 \pm 0.68 \mathrm{~mm}$, respectively. The mean increase in width was 2.92 $\pm 0.89 \mathrm{~mm}(P<0.001)$.

\section{Conclusion:}

This non-invasive and simple technique provided an acceptable increase in ridge width. As the sample was small, we recommend further clinical investigation with larger samples to confirm that this technique may be used successfully as an alternative to current invasive augmentation methods.

Keywords: Augmentation, autogenous bone, bone graft, implant.

\section{INTRODUCTION}

Endosseous implants have become a trusted treatment modality for completely or partially edentulous patients. In recent years, patients and doctors have become more aware of the favorable aesthetic and functional characteristics of implants, which necessitate implant placement in the prosthetic, rather than surgical, position. Prosthetic implantation requires the presence of sufficient high-quality bone.

\footnotetext{
* Address correspondence to this author at the Department of Periodontics, Dental School, Mashhad University of Medical Sciences, Vakilabad Blvd., Mashhad, Iran; Tel: 0098511 6012189; Fax: 0098511 8829500; E-mail: Moeentaghavia@mums.ac.ir
} 
The presence of teeth preserves the alveolar processes of the maxilla and mandible, and natural bone resorption begins following tooth loss. Severe resorption may prohibit implant placement in the prosthetic position, necessitating a ridge augmentation procedure to obtain sufficient bone [1]. Several previous studies have evaluated the success of implant placement after ridge augmentation [2 - 4]. Various techniques have been introduced for lateral ridge augmentation, as a procedure performed simultaneously with implantation or as a separate surgery [5 - 7]. Although these techniques have had high success rates, they are usually invasive and expensive. Autografts harvested intra- or extraorally, in block or particulated form, have been recommended as the best materials for this procedure specially for limited ridge augmentations. They can be obtained in a number of ways and from several donor sites. In an animal study, Von Arx et al. observed greater ridge enlargement (horizontal bone gain) at autograft + expanded polytetrafluoroethylene sites than at sites prepared with other materials [8]. Maiorana et al. covered autogenous corticocancellous onlay graft with a thin layer of anorganic bovine bone granules and collagen membrane to reduce graft resorbtion. Histomorphometric analaysis showed proposed technique was able to maintain the original bone volume of the blocks [9].



Fig. (1). Trephine drills used to harvest autogenous bone.

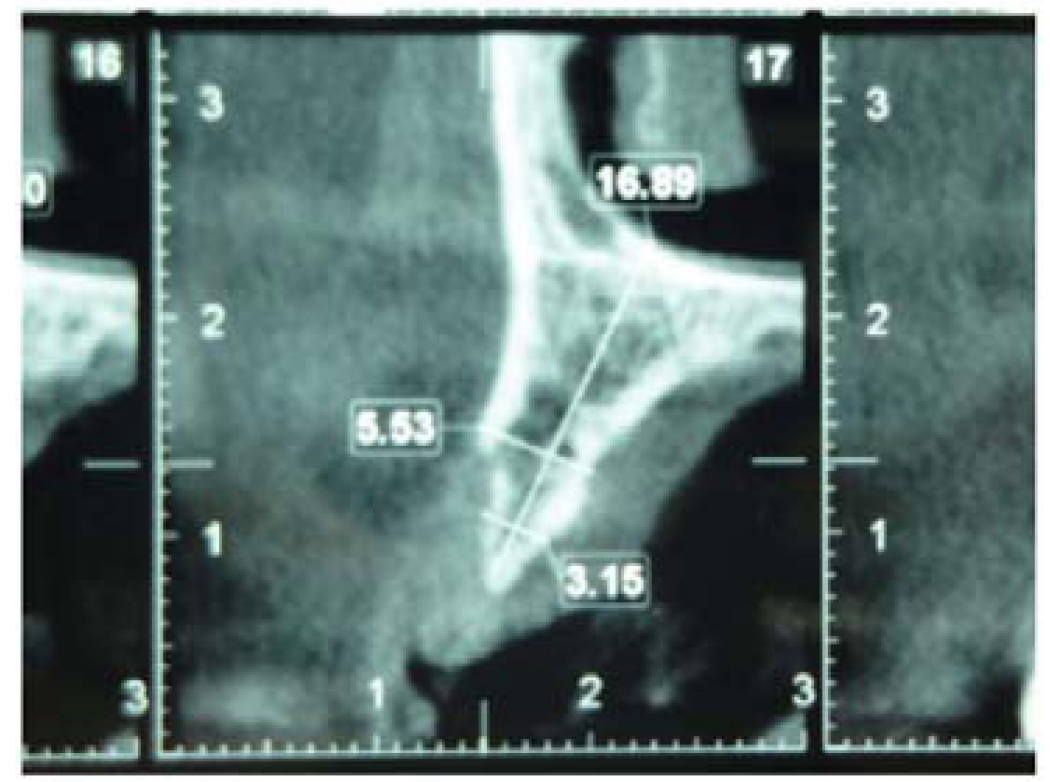

Fig. (2). Cone beam computed tomographic image of the recipient site before surgery.

Laino et al. studied bone formation in atrophic posterior mandibles augmented by autologous bone block harvested from chin and compared this technique with corticocancellous bone block allograft. Histological analysis showed no 
statistically significant differences between the two groups regarding the percentage of newly formed bone [10].In an examination of donor-site morbidity in bone harvesting from the iliac crest, Cricchio and Lundgren observed a high success rate but prolonged complications in more than $25 \%$ of patients. Steigmann et al. proposed periosteal pocket flap for horizontal bone regeneration and reported periosteal pocket flap design could be a predictable alternative flap approach for correction of severe or localized horizontal bone deficiencies [11].

The use of growth factors such as recombinant human bone morphogenic protein-2 (rhBMP-2) impregnated with absorbable collagen sponge and titanium mesh showed good result in treating of large bone defects [12].

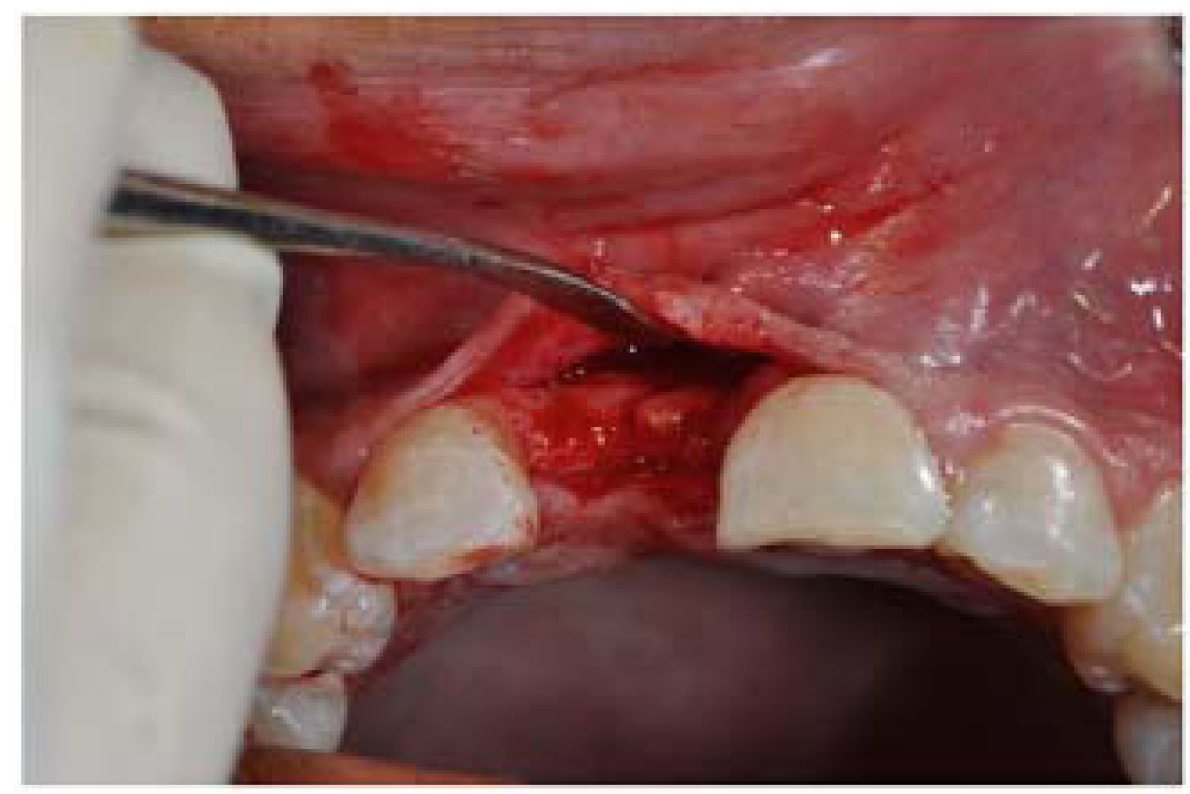

Fig. (3). Crestal incision and insufficient bone at the recipient site.

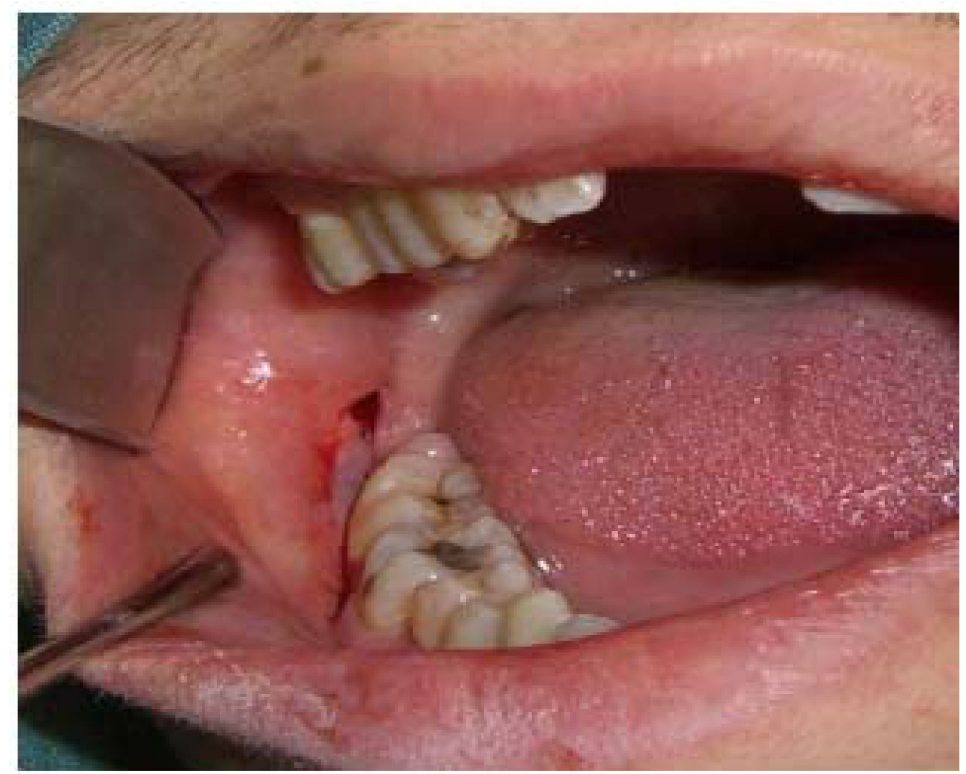

Fig. (4). Incision at the donor site.

Cicciue et al. developed a new bone technique reconstruction not involving autogenous bone graft. they used rhBMP-2 with carrier consisted of an absorbable collagen sponge and reported excellent newly formed bone of treated area [13].

Because routine ridge augmentation techniques are complicated and the materials are expensive and require lengthy 
healing periods, we aimed in this pilot study to evaluate changes in the alveolar ridge following a new chairside ridge augmentation procedure using autografts harvested with trephine drills and placed without screws.

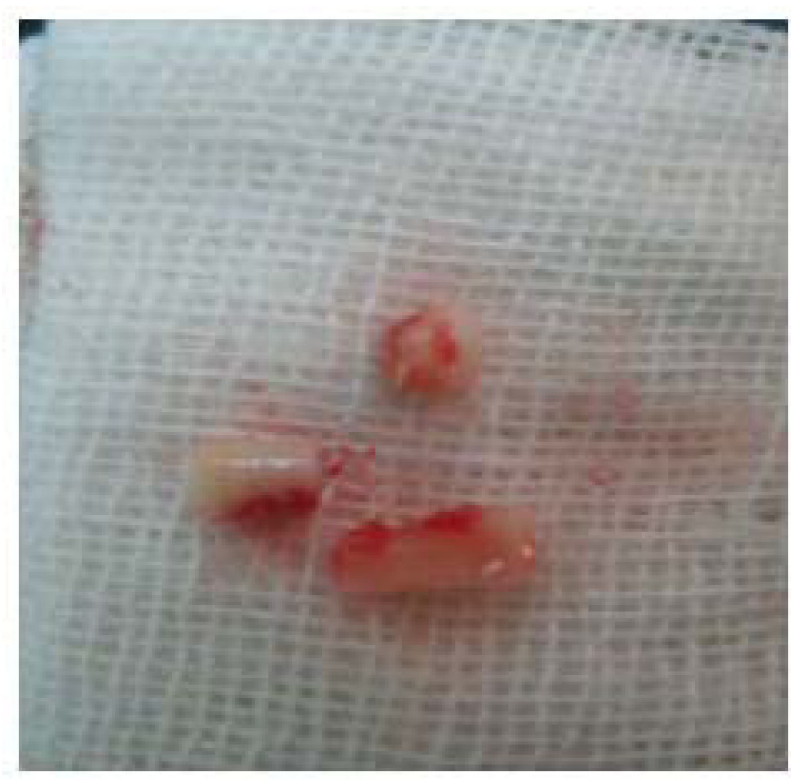

Fig. (5). Harvest of bone blocks from the external oblique ridge.

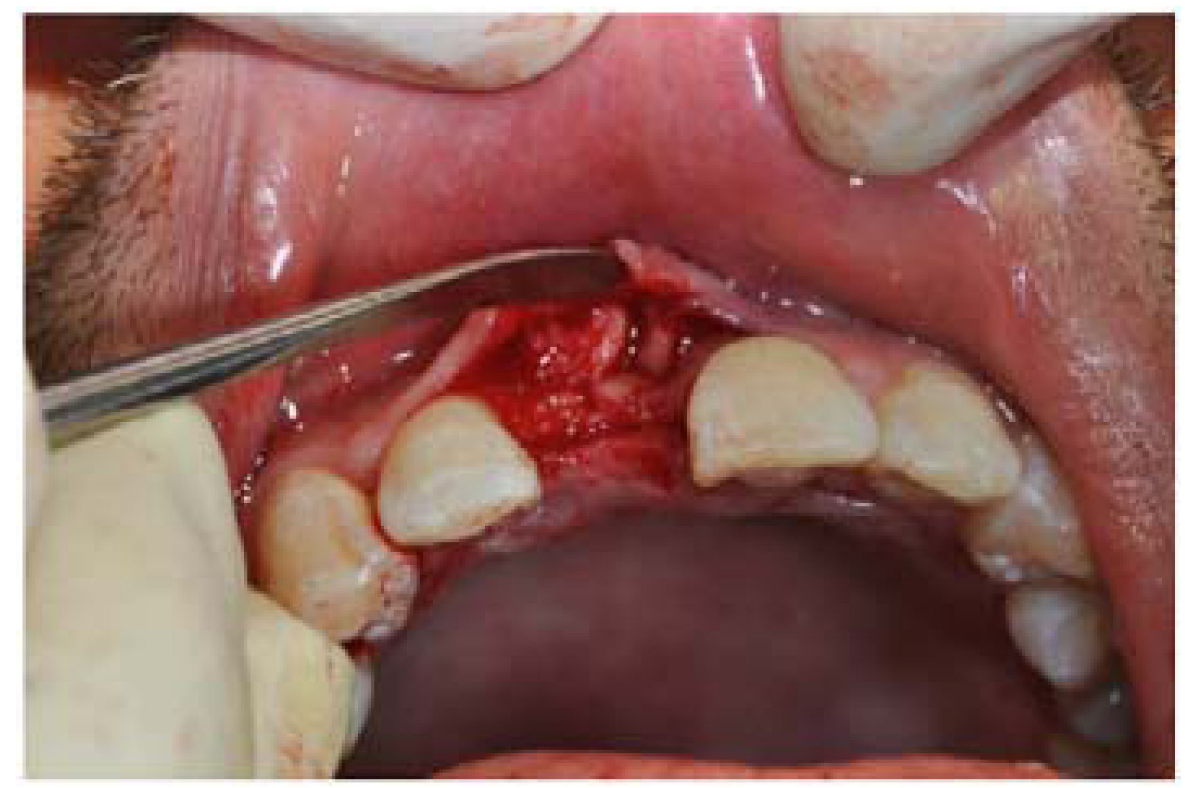

Fig. (6). Placement of bone blocks at the recipient site.

\section{MATERIALS AND METHODS}

\section{Patient Selection}

This "before and after" quasi-experimental interventional study involved 30 patients referred to the Department of Implantology, Mashhad Dental School, Iran. The institutional review board of Mashhad Dental School and the ethical review board of the Mashad University of Medical Sciences approved this study, and all participants provided written informed consent. The medical protocol and ethical considerations followed the guidelines of the Declaration of Helsinki. The study protocol was registered in the Iran Registry of Clinical Trials (www.irct.ir; ID: IRCT138902201601N3). Selection criteria were: 1) lack of contra-indication to dental implant placement or oral surgery; 2) suitable occlusal relationships at the implant placement site; 3) lack of periodontal disease or infection in 
any other teeth, especially around the treatment site; 4) sufficient bone height but insufficient width at the treatment site; and 5) absence of a single tooth.

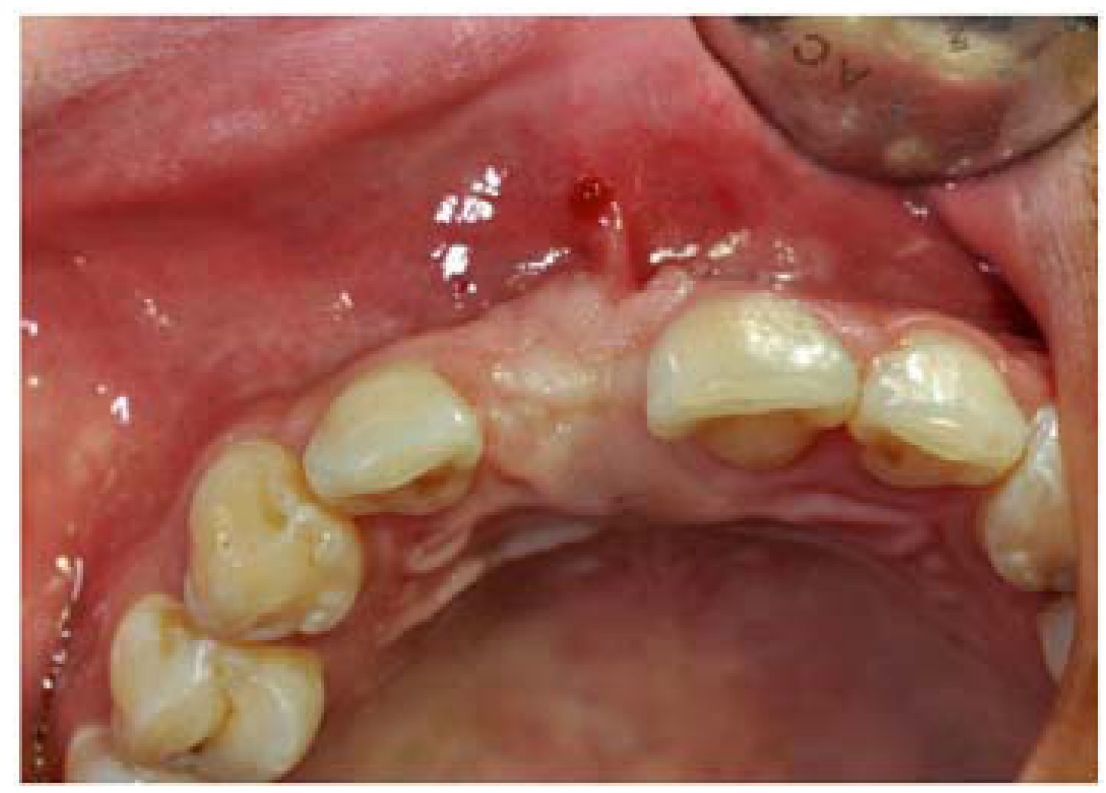

Fig. (7). Recipient site 3 months after surgery.

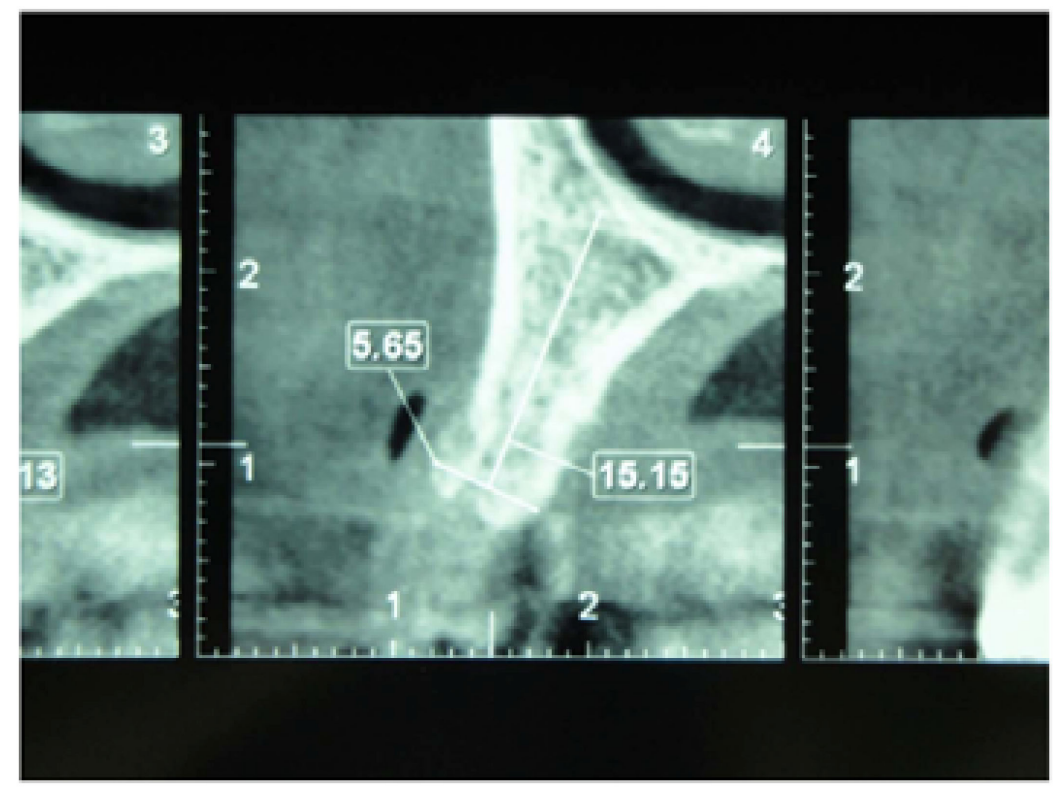

Fig. (8). Cone beam computed tomographic image of the recipient site 3 months postoperatively.

\section{Surgical Procedure}

In the present study, the recipient sites were limited in size, and trephine drills (Fig. 1) were used to obtain bone grafts. Primary evaluations were performed by cone beam computed tomography (CBCT; Fig. 2). After anesthetizing the operation site with lidocaine (1:100000; Daroopakhsh,Tehran, Iran), a crestal incision was performed. A second practitioner measured bone width. An envelope flap was retracted using blunt dissection to limit it to the graft site, with no excessive extension (Fig. 3). The periosteum was raised, intact and undamaged, from the bone. The flap was extended laterally to obtain sufficient space for the bone graft, and no vertical incision was made. Bone was obtained from the external oblique ridge area. A buccal sulcular incision was made to expose the donor site from the distal end of the first molar to the distal aspect of the second (or third, if present) molar (Fig. 4). A \#5 or \#6 trephine drill (Hager \& Meisinger GmbH, Neuss, Germany) was used as needed to harvest one or two pieces of bone (Fig. 5). 
Next, the donor site was sutured and compressed with damp gauze sponge. The bone blocks were placed inside the envelope flap at the recipient site (Fig. 6). In some cases, the shape of the bone graft was adjusted to enhance its adaptation to the recipient site. Due to the limited extension of the flap and the orderly setting of the bone blocks, the use of screws to secure the bone grafts in place was not necessary.

The recipient site was then sutured and covered with periodontal dressing (Coe-pak; GC America,IL, USA). From $24 \mathrm{~h}$ prior to the operation, patients were administered amoxicillin $(500 \mathrm{mg})$ every $6 \mathrm{~h}$, and the treatment continued for 5-7 days. Chlorhexidine mouthwash ( $0.2 \%$ solution) was prescribed for 2 weeks to all patients. Patients were also prescribed acetaminophen plus codeine one tablet each 4-6 hours(300 mg Acetaminophen +10 mg Codeine phosphate, Abidi Co, Tehran, Iran). Sutures were removed 1 week postoperatively. Three months later, another CBCT examination was performed, the recipient site was reopened, bone width was measured (Figs. 7-9), and the implants were placed (Fig. 10).

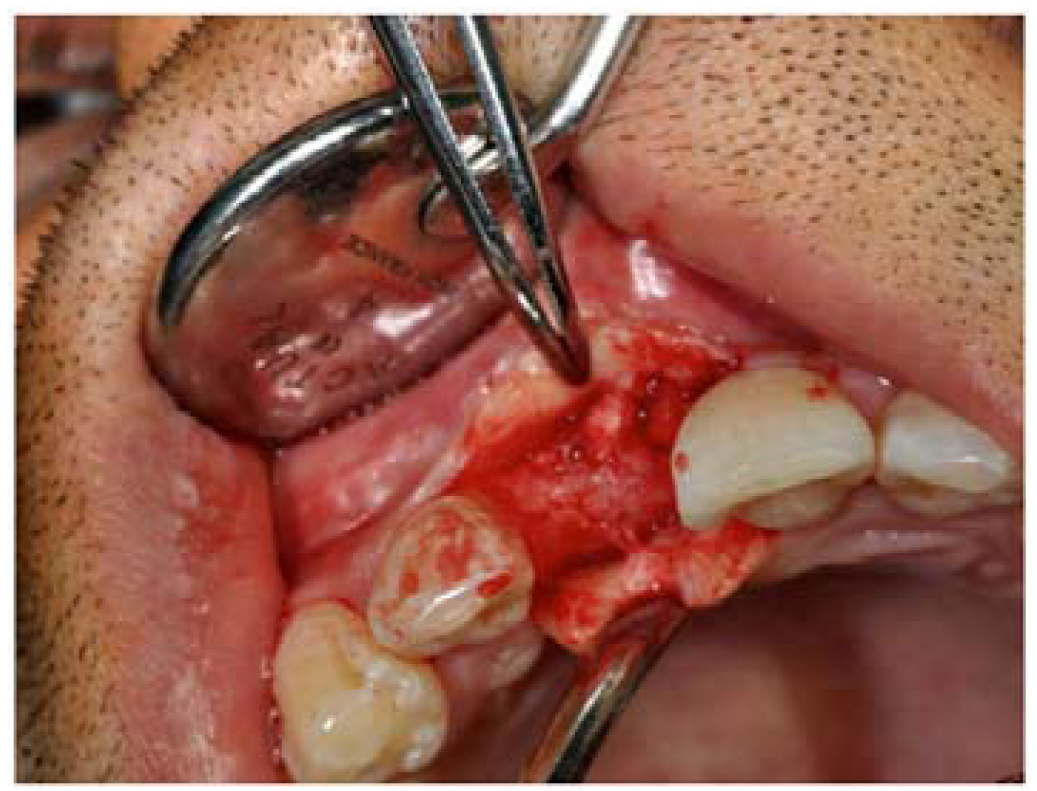

Fig. (9). Augmented ridge.

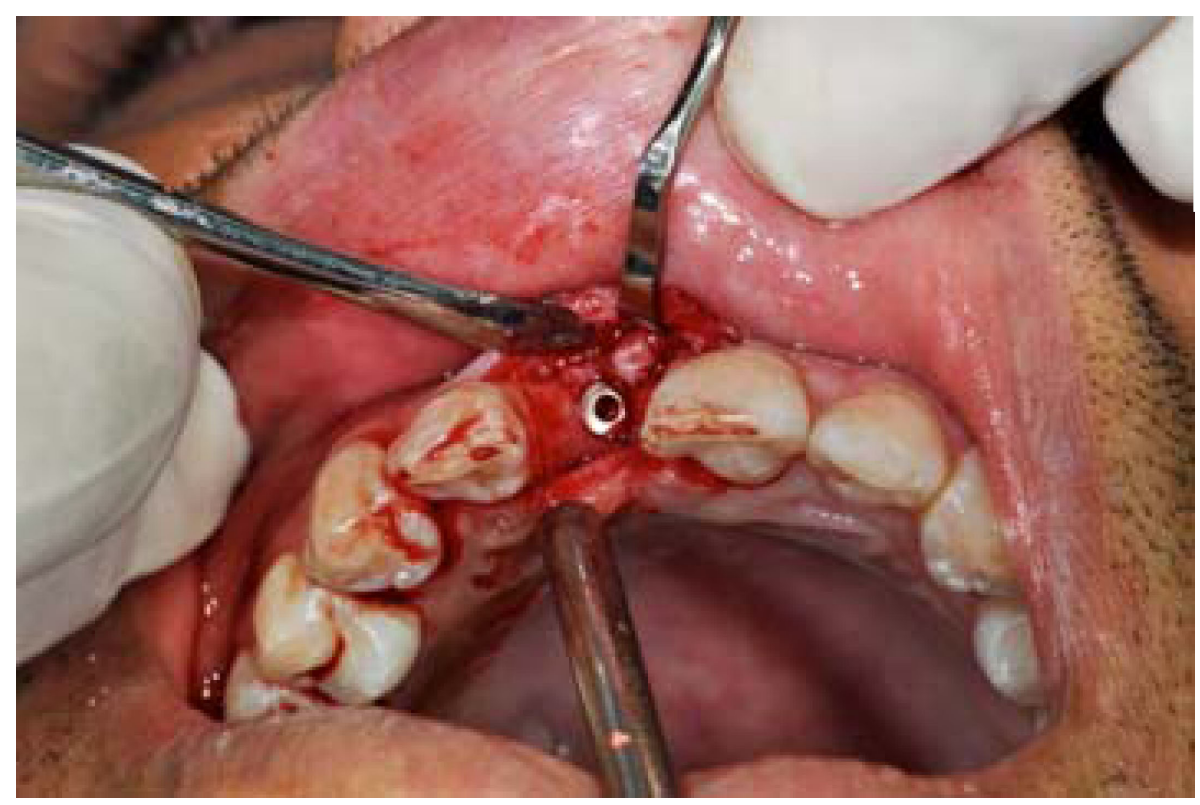

Fig. (10). Implant placement. 


\section{Statistical Analysis}

The Kolmogorov-Smirnov test was used to evaluate the normality of data. As data distribution was normal, the paired $t$-test was used to assess mean changes in bone width.

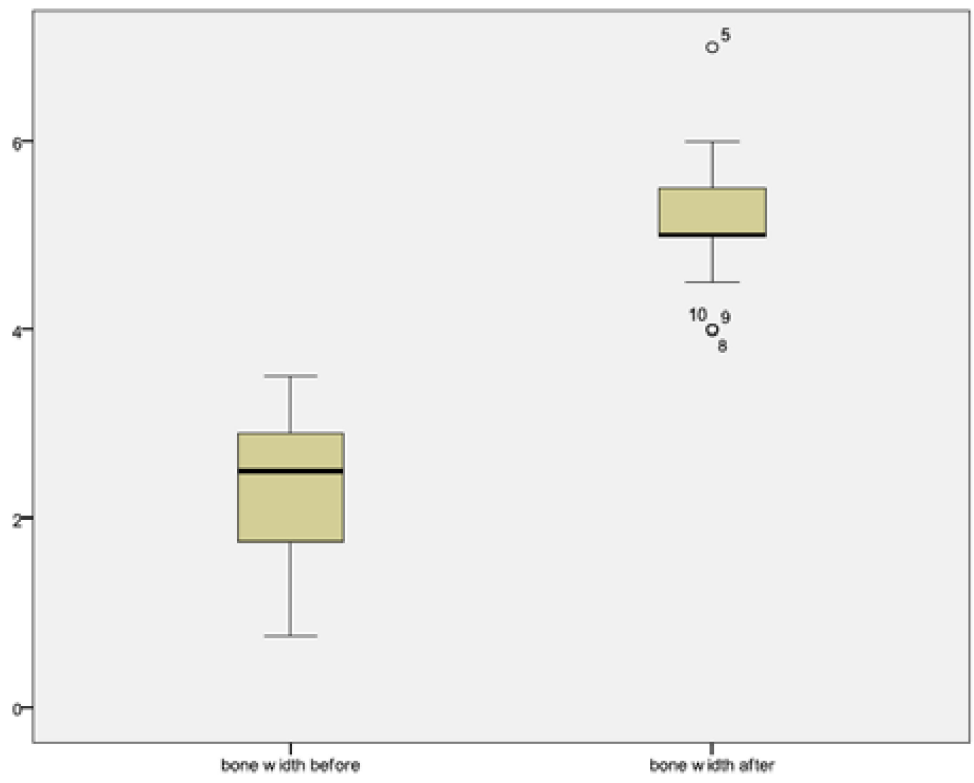

Fig. (11). Box plot showing bone width before and after augmentation.

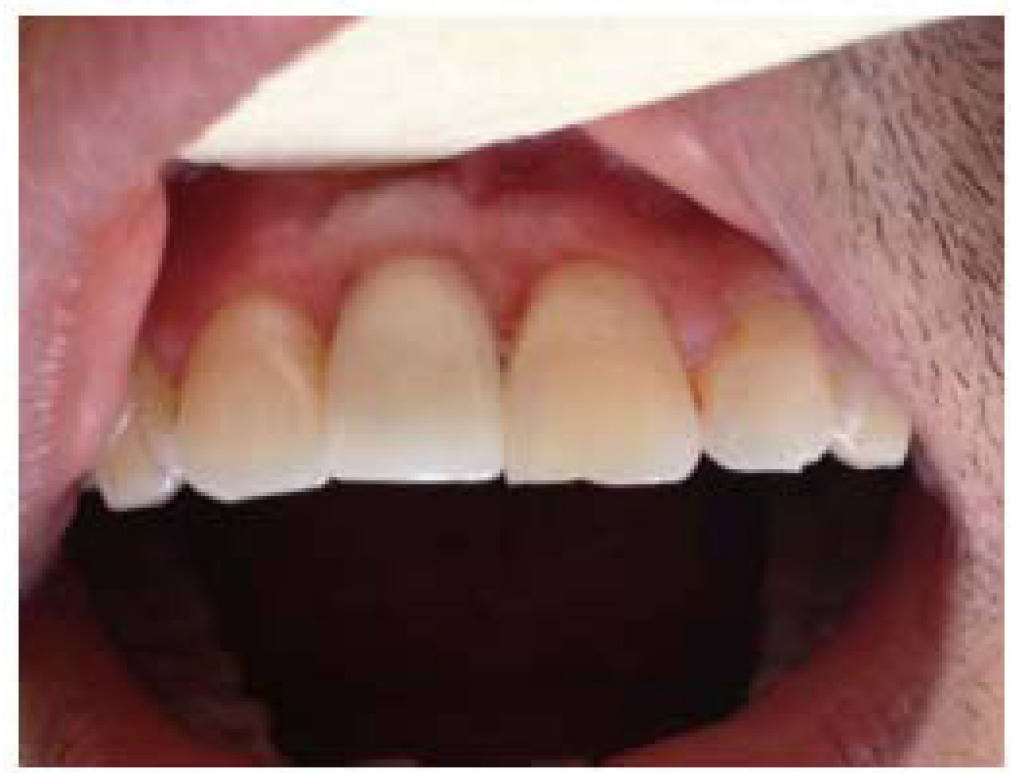

Fig. (12). Same patient after 6 years.

\section{RESULTS}

The study sample comprised 17 women and 13 men (mean age, $45.6 \pm 5.3$ years). Five patients were excluded from the study due to graft failure ( $n=2$; one man and one woman) or refusal to continue the study $(n=3)$. The results of the remaining 25 patients were analyzed. Table 1 shows pre- and post-treatment bone widths. The mean preoperative and postoperative ridge widths were $2.23 \pm 0.79 \mathrm{~mm}$ and $5.16 \pm 0.68 \mathrm{~mm}$, respectively. The mean increase in ridge width $(2.92 \pm 0.89 \mathrm{~mm})$ was significant $(P<.001, t=16.383$; Table 2, Fig. 11).

In cases in which the periosteum was damaged during flap elevation, the bone grafts were partially resorbed. However, We were able to place implants after 3 months in all cases. 
Figs. (12 and 13) show different patients treated with this technique. As this was a pilot study and we did not calculate the adequate sample size, we calculated the power of our finding and it was $85 \%$.

Table 1. Pre- and postoperative volumetric data for all patients.

\begin{tabular}{|c|c|c|}
\hline Patient & Preoperative bone width $(\mathrm{mm})$ & Postoperative bone width $(\mathrm{mm})$ \\
\hline 1 & 1 & 5.5 \\
\hline 2 & 2.5 & 5.5 \\
\hline 3 & 2.9 & 5 \\
\hline 4 & 0.75 & 5 \\
\hline 5 & 3 & 7 \\
\hline 6 & 1.5 & 6 \\
\hline 7 & 2 & 5 \\
\hline 8 & 2.9 & 4 \\
\hline 9 & 2 & 4 \\
\hline 10 & 2 & 45 \\
\hline 11 & 1.5 & 5 \\
\hline 12 & 1 & 4.5 \\
\hline 13 & 3.5 & 5.5 \\
\hline 14 & 2.5 & 5 \\
\hline 15 & 3 & 5.5 \\
\hline 16 & 2.5 & 5 \\
\hline 17 & 2.5 & 5.5 \\
\hline 18 & 2 & 4.5 \\
\hline 19 & 3 & 5.5 \\
\hline 20 & 3 & 5.5 \\
\hline 21 & 1 & 5.5 \\
\hline 22 & 2 & 4.5 \\
\hline 23 & 1.75 & 5 \\
\hline 24 & 2.5 & 5.5 \\
\hline 25 & 3.5 & 6 \\
\hline
\end{tabular}

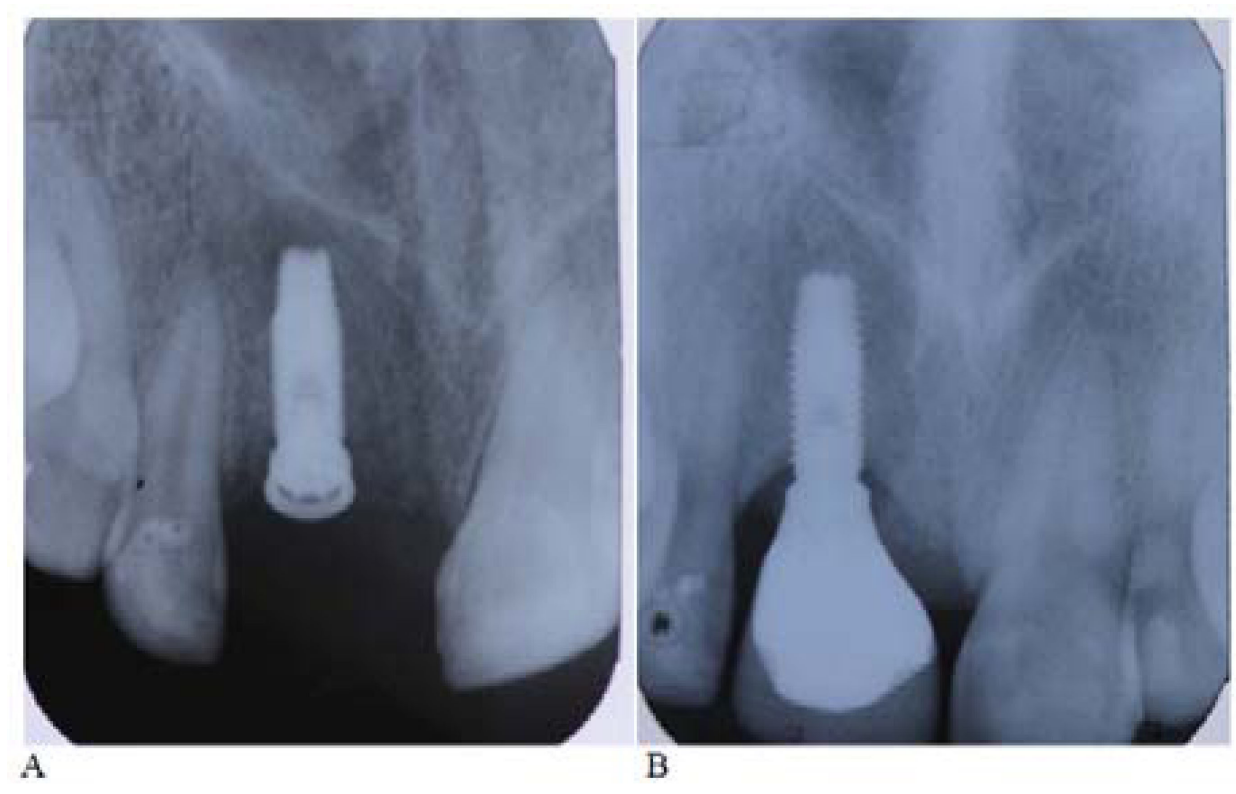

Fig. (13). Radiographic images of the patient at baseline (A) and after 6 years (B).

\section{DISCUSSION}

Titanium endosseous implants have become a successful treatment for tooth loss and are predictably associated with 
a good prognosis. In some cases, implant placement is difficult or even impossible due to bone resorption. In such situations, the use of bone grafts, especially autogenous grafts, is an excellent solution with many advantages.

Autografts are considered to be the gold standard for bone transplantation because they are osteogenic, osteoconductive, and osteoinductive [14]. Autografts can be taken from an extraoral (e.g., eggs, iliac crest) or intraoral (e.g., chin or ramus) source. Although they are highly advantageous due to a minimal or no risk of infectious disease transmission [15] and lack of immune response after grafting [16], routine autogenous bone harvesting techniques (especially those at extraoral sites) are associated with risks such as donor-site morbidity, limited bone availability, drooping of the chin, paresthesia due to nerve damage, tooth devitalization, gingival recession, increased postoperative discomfort, infection, and blood loss [17].

However, one disadvantage of autogenous bone grafts is that they require two simultaneous surgeries. This study was performed to evaluate a simple method for ridge augmentation in limited edentulous areas involving the harvest of bone grafts from the external oblique ridge. Verdugoe et al. evaluated bone graft donor sites in different areas of the mandible and found that the second and third molar regions could provide high-quality bone with a mean thickness of $2.8 \pm 0.6 \mathrm{~mm}$ [18]. The technique described in the present study was a simple, non-aggressive operation beginning with the elevation of an envelope flap limited to the edentulous area. Bone blocks harvested with trephine drills were placed into the envelope, with no need for fixation.

In routine procedures, the recipient site is decorticated to enhance the blood supply. Such decortication is not necessary with the novel method described here because the bone blocks are small. If the periosteum remains undisturbed, it can provide sufficient nutrients for the graft. As it was mentioned before, in cases of periostal damage during flap elevation, the bone grafts were resorbed.

We think that the simplicity and the lack of a need for recipient site decortication or screw fixation in this technique, are its main advantages. The significant increase in mean bone width observed in this study was comparable to the results of studies which have been performed on the other more difficult techniques. For instance, Acocella et al. (2009) used bone blocks obtained from the mandibular ramus for lateral augmentation and reported a $4 \pm 0.77 \mathrm{~mm}$ increase in ridge width [2]. Funaki et al. used distraction osteogenesis and bisection techniques to increase ridge width by 2.7 and $1.7 \mathrm{~mm}$, respectively [19]. Another advantage of this technique is the shorter time required for complete healing. We were able to place implants after 3 months in all cases, whereas a healing period of at least 4-5 months before implant placement is recommended following other graft techniques [20, 21]. Rapid vascularization of the bone block is essential for successful remodeling and new bone formation [22].Revascularization of cortical transplants begins 6 days after graft placement and is completed in 1-2 months $[23,24]$. The short healing time observed in this study seems sensible, as the bone blocks were small.

This study has several limitations. First, the sample was small, although the power of the results was $92 \%$. Second, because we were attempting to prove the principles of this easy technique, we did not perform comparison with another group; like conventional grafting using fixed bone graft to such analysis should be conducted in the context of a larger trial. Lack of graft fixation using screws, can be considered as the main advantage because it simplifies this technique, on the other hand it might have some deleterious effects on bone healing and undesired consequences due to graft movement. We used a limited envelope flap to retain the grafted blocks in proper position

Table 2. Comparison of pre- and post-operation bone width.

\begin{tabular}{|c|c|c|c|c|c|}
\hline Ridge width & Number of samples & $\begin{array}{l}\text { Mean } \\
(\mathbf{m m})\end{array}$ & SD & T & Pv \\
\hline Pre-operation & 25 & 2.232 & 0.7945 & \multirow{2}{*}{16.383} & 0.000 \\
\cline { 1 - 4 } & 25 & 5.160 & 0.6879 & & \\
\hline Post-operation & 25 & 2.928 & 0.8936 & \\
\hline
\end{tabular}

\section{CONCLUSION}

This pilot study demonstrated that this non-invasive and simple method provides an acceptable increase in ridge width. As our sample was small, we recommend further clinical investigation with larger samples to confirm that this technique may be used successfully as an alternative to current, invasive augmentation methods. Future clinical trials should also be conducted to compare the outcomes obtained with this method to those achieved with routine invasive techniques. 


\section{CONFLICT OF INTEREST}

The authors confirm that this article content has no conflict of interest.

\section{ACKNOWLEDGEMENTS}

This study was supported by a grant from the Research Council of Mashhad University of Medical Sciences.

\section{REFERENCES}

[1] Fu JH, Wang HL. Horizontal bone augmentation: the decision tree. Int J Periodontics Restorative Dent 2011; 31(4): $429-36$.

[2] Acocella A, Bertolai R, Colafranceschi M, Sacco R. Clinical, histological and histomorphometric evaluation of the healing of mandibular ramus bone block grafts for alveolar ridge augmentation before implant placement. J Craniomaxillofac Surg 2010; 38(3): 222-30. [http://dx.doi.org/10.1016/j.jcms.2009.07.004]

[3] Misch CM, Misch CE, Resnik RR, Ismail YH. Reconstruction of maxillary alveolar defects with mandibular symphysis grafts for dental implants: a preliminary procedural report. Int J Oral Maxillofac Implants 1992; 7(3): 360-6.

[4] Zahrani AA. Augmentation in two stages of atrophic alveolar bone prior to dental rehabilitation: a case report. J Contemp Dent Pract 2007; 8(6): 57-63.

[5] Donos N, Mardas N, Chadha V. Clinical outcomes of implants following lateral bone augmentation: systematic assessment of available options (barrier membranes, bone grafts, split osteotomy). J Clin Periodontol 2008; 35(Suppl 8): $173-202$. [http://dx.doi.org/10.1111/j.1600-051X.2008.01269.x]

[6] Urban IA, Nagursky H, Lozada JL. Horizontal ridge augmentation with a resorbable membrane and particulated autogenous bone with or without anorganic bovine bone-derived mineral: a prospective case series in 22 patients. Int J Oral Maxillofac Implants 2011; 26(2): 404-14.

[7] Yeo A, Cheok C, Teoh SH, Zhang ZY, Buser D, Bosshardt DD. Lateral ridge augmentation using a PCL-TCP scaffold in a clinically relevant but challenging micropig model. Clin Oral Implants Res 2012; 23(12): 1322-32. [http://dx.doi.org/10.1111/j.1600-0501.2011.02366.x]

[8] von Arx T, Cochran DL, Schenk RK, Buser D. Evaluation of a prototype trilayer membrane (PTLM) for lateral ridge augmentation: an experimental study in the canine mandible. Int J Oral Maxillofac Surg 2002; 31(2): 190-9. [http://dx.doi.org/10.1054/ijom.2001.0205]

[9] Maiorana C, Beretta M, Battista GG, et al. Histomorphometric evaluation of anorganic bovine bone coverage to reduce autogenous grafts resorption: preliminary results. Open Dent J 2011; 5: 71-8. [http://dx.doi.org/10.2174/1874210601105010071]

[10] Laino L, Iezzi G, Piattelli A, Lo Muzio L, Cicciu M. Vertical ridge augmentation of the atrophic posterior mandible with sandwich technique: bone block from the chin area versus corticocancellous bone block allograft: clinical and histological prospective randomized controlled study. BioMed Res Int 2014; 2014; 982104.

[11] Cricchio G, Lundgren S. Donor site morbidity in two different approaches to anterior iliac crest bone harvesting. Clin Implant Dent Relat Res 2003; 5(3): 161-9. [http://dx.doi.org/10.1111/j.1708-8208.2003.tb00198.x]

[12] Cicciu M, Herford AS, Stoffella E, Cervino G, Cicciu D. Protein-signaled guided bone regeneration using titanium mesh and rh-BMP2 in oral surgery: A case report involving left mandibular reconstruction after tumor resection. Open Dent J 2012; 6: 51-5. [http://dx.doi.org/10.2174/1874210601206010051]

[13] Cicciu M, Herford AS, Cicciu D, Tandon R, Maiorana C. Recombinant human bone morphogenetic protein-2 promote and stabilize hard and soft tissue healing for large mandibular new bone reconstruction defects. J Craniofac Surg 2014; 25(3): 860-2. [http://dx.doi.org/10.1097/SCS.0000000000000830]

[14] Rosenberg E, Rose LF. Biologic and clinical considerations for autografts and allografts in periodontal regeneration therapy. Dent Clin North Am 1998; 42(3): 467-90.

[15] Goldberg VM, Stevenson S. Natural history of autografts and allografts. Clin Orthop Relat Res 1987; (225): 7-16.

[16] Schlegel KA, Fichtner G, Schultze-Mosgau S, Wiltfang J. Histologic findings in sinus augmentation with autogenous bone chips versus a bovine bone substitute. Int J Oral Maxillofac Implants 2003; 18(1): 53-8.

[17] Mellonig JT. Autogenous and allogeneic bone grafts in periodontal therapy. Critical reviews in oral biology and medicine: an official publication. Am Assoc Oral Biol 1992; 3(4): 333-52.

[18] Verdugo F, Simonian K, Smith McDonald R, Nowzari H. Quantitation of mandibular ramus volume as a source of bone grafting. Clin Implant Dent Relat Res 2009; 11(Suppl. 1): e32-7. [http://dx.doi.org/10.1111/j.1708-8208.2009.00172.x]

[19] Funaki K, Takahashi T, Yamuchi K. Horizontal alveolar ridge augmentation using distraction osteogenesis: comparison with a bone-splitting method in a dog model. Oral Surg Oral Med Oral Pathol Oral Radiol Endod 2009; 107(3): 350-8. [http://dx.doi.org/10.1016/j.tripleo.2008.10.005]

[20] McAllister BS, Haghighat K. Bone augmentation techniques. J Periodontol 2007; 78(3): 377-96. 
[http://dx.doi.org/10.1902/jop.2007.060048]

[21] Pikos MA. Mandibular block autografts for alveolar ridge augmentation. Atlas Oral Maxillofac Surg Clin North Am 2005; 13(2): 91-107. [http://dx.doi.org/10.1016/j.cxom.2005.05.003]

[22] Albrektsson T. Repair of bone grafts. A vital microscopic and histological investigation in the rabbit. Scand J Plast Reconstr Surg 1980; 14(1): $1-12$. [http://dx.doi.org/10.3109/02844318009105731]

[23] Fonseca RJ, Clark PJ, Burkes EJ Jr, Baker RD. Revascularization and healing of onlay particulate autologous bone grafts in primates. J Oral Surg 1980; 38(8): 572-7.

[24] Kusiak JF, Zins JE, Whitaker LA. The early revascularization of membranous bone. Plast Reconstr Surg 1985; 76(4): 510-6. [http://dx.doi.org/10.1097/00006534-198510000-00003]

Received: December 25, 2014

Revised: November 9, 2015

Accepted: November 10, 2015

(c) Arab et al. ; Licensee Bentham Open.

This is an open access article licensed under the terms of the Creative Commons Attribution-Non-Commercial 4.0 International Public License (CC BY-NC 4.0) (https://creativecommons.org/licenses/by-nc/4.0/legalcode), which permits unrestricted, non-commercial use, distribution and reproduction in any medium, provided the work is properly cited. 